\title{
Charged three-body system with arbitrary masses near conformal invariance
}

\author{
A. Delfino, ${ }^{1}$ T. Frederico, ${ }^{2}$ and Lauro Tomio ${ }^{3,4}$ \\ ${ }^{1}$ Instituto de Física, Universidade Federal Fluminense, 24210-900 Niterói, RJ, Brazil \\ ${ }^{2}$ Departamento de Física, Instituto Tecnológico de Aeronáutica, 12228-900 São José dos Campos, SP, Brazil \\ ${ }^{3}$ Instituto de Física Teórica, Universidade Estadual Paulista (UNESP), 01140-070 São Paulo, SP, Brazil \\ ${ }^{4}$ Centro Brasileiro de Pesquisas Físicas, Rua Dr. Xavier Sigaud, 150, 22290-180 Rio de Janeiro, RJ, Brazil
}

(Received 10 June 2009; published 30 November 2009)

\begin{abstract}
Within an adiabatic approximation to the three-body Coulomb system, we study the strength of the leading-order conformaly invariant attractive dipole interaction produced when a slow charged particle $q_{3}$ (with mass $m_{3}$ ) is captured by the first-excited state of a dimer [with individual masses and charges $\left(m_{1}, q_{1}\right)$ and $\left.\left(m_{2}, q_{2}=-q_{1}\right)\right]$. The approach leads to a universal mass-charge critical condition for the existence of three-body level condensation, $\left(m_{1}^{-1}+m_{2}^{-1}\right) /\left[\left(m_{1}+m_{2}\right)^{-1}+m_{3}^{-1}\right]>\left|q_{1} /\left(24 q_{3}\right)\right|$, as well as the ratio between the geometrically scaled energy levels. The resulting expressions can be relevant in the analysis of recent experimental setups with charged three-body systems, such as the interactions of excitons, or other matter-antimatter dimers, with a slow charged particle.
\end{abstract}

DOI: $10.1103 /$ PhysRevA.80.052509

PACS number(s): 36.10.-k, 03.65.Ge

\section{INTRODUCTION}

In view of the actual experimental possibilities, we recall some general characteristics of three-body charged systems with arbitrary masses and two different charges, in order to derive the charge-mass dependence of the leading-order strength of the attractive dipole interaction produced by a bound two-body subsystem (with individual charges $q_{1}$ and $\left.q_{2}=-q_{1}\right)$ in the third particle with charge $q_{3}$. We start our investigation by considering the old and well-known case of the interaction of a positronium $(\mathrm{Ps}$, a bound state of an electron $e^{-} \equiv-e$ and a positron $e^{+} \equiv+e$, where $e$ is the absolute value of the electron charge) with a spectator electron. This is the ionized negative positronium $\left(\mathrm{Ps}^{-}\right)$. Early calculations, by Wheeler in 1946 [1], have already predicted a bound state for such system, confirmed by Mills in 1981 [2].

Further investigations on the properties of $e^{-} e^{+} e^{-}$system [3-6], as well as on other Coulombic three-body systems, since 1960s up to recent years [7-11], have also been motivated by the increasing interest in matter-antimatter interactions [12]. Up to 1995 , the theoretical and experimental advances in understanding Coulombic three-body systems and matter-antimatter interaction can be found, respectively, in two reviews $[10,12]$. As emphasized in [12], small number of leptons, electrons, muons and their antiparticles, are important to test fundamental theories of quantum electrodynamics; and systems with small number of protons and antiprotons can also provide relevant tests of the strong interaction. The actual interest on the properties of few-body charged systems is evidenced by the recent report on the production of a molecular dipositronium $\mathrm{Ps}_{2}$ [13]. For a recent review, particularly concerned on the stability of quantum charged few-body systems, see [11]. In Ref. [4], Rost and Wintgen explored and classified the $\mathrm{Ps}^{-}$dynamics considering the observation that it has a molecular structure similar to the ionized hydrogen molecule $H_{2}^{+}\left[\left(p e^{-}\right) p\right]$. They have also reported the existence of a ${ }^{1} S$ resonance pattern unknown in threebody Coulomb systems. Such results, combined with results obtained for the hydrogen ion $H^{-}\left[\left(p e^{-}\right) e^{-}\right]$, lead them to sug- gest the existence of a similar resonance spectrum for all $A B A$ Coulomb systems with charge and mass ratios such that $\left|q_{A} / q_{B}\right|=1$ and $m_{A} / m_{B} \geq 1$, respectively. More recently, by considering a molecular adiabatic (MA) treatment for the $\mathrm{Ps}^{-}$, it was also reported an accumulation of three-body resonances above the two-body threshold [5].

Surprisingly, besides the number of recent studies concerned with few-charged quantum systems [11], and the recognized relevance of a mass-charge universal relation in view of actual experimental facilities [4], a particular straightforward condition relating charges and masses for a general three-body system (with arbitrary masses) is still missing in the literature. In the case of exotic molecular three-body systems, where the subsystem is hydrogenlike (with $e^{-}$replaced by $\mu^{-}$or $\pi^{-}$), it was shown in [14] that a relation for the structure of the spectrum can be obtained in the frame of Born-Oppenheimer approximation, following spectral properties of long-ranged $1 / R^{2}$ interactions [15], which are known to be conformally invariant $[16,17]$.

The study of resonance patterns, which can occur in fewbody interactions, became very relevant in trapped ultracold atom experiments, as the presence of several resonances at experimentally accessible magnetic fields can allow precise tuning of atom-ion interaction [18]. Theoretical predictions such as the increasing number of three-body bound states when the two-body scattering length goes to infinity, known as Efimov effect [19], can actually be checked experimentally in ultracold atomic laboratories [20]. The spectrum of Efimov states, exhibiting a geometrical scaling, is generated by an attractive effective potential proportional to $1 / R^{2}$, where $R$ is the distance of one of the particles to the center of mass of the remaining pair, considering short-range twobody interactions [19,21] (on the scaling mechanism and conformal invariance behind this effect, see $[16,17,22])$. As it will be shown, the long-ranged Coulombic interactions, for certain configurations of three-charged particles, can exhibit the same kind of effective interaction.

For recent relevant applications in ultracold laboratories, of a study with three-charged systems, we can mention the possibility of exciton $[23,24]$ or positronium $[25,26]$ con- 
densed gas interacting with a charged particle. In case of excitons (electron-hole bound pair in a semiconductor), the electron and hole in the interacting pair can acquire effective individual masses distinct from the free-electron mass [24]. When interacting with a slow charged particle, a charge-mass dependent resonance pattern should emerge. So, well-based simple charge-mass conditions can be very helpful to analyze the relation between effective masses and the observed spectrum.

Motivated by the above discussion we show examples in atomic physics of charged three-body systems in which appears an effective long-range $1 / R^{2}$ potential, where the strength is modulated by the arbitrary individual masses and charges, restricted to the condition that the bound subsystem is neutral. A robust mass-charge critical condition is derived for the weakly bound three-body spectrum below the state $n=2$ of the subsystem, in case of arbitrary masses. Here, we should observe that the degeneracy between the $2 S$ and $2 P$ levels of the chargeless two-body subsystem is broken by the dipole potential. When it is attractive it rises to the spectrum below [4] that we consider; the repulsive part is responsible for a set of resonances above the $2 S-2 P$ state [5]. The approach is strictly valid for the cases where the dimer is made up with pointlike charged particles. However, it can also be taken as a first approach when the charged particles are more complex objects.

The basic equations of our formalism, in the adiabatic approximation, are given in the next section, which lead to the effective $1 / R^{2}$ long-range interaction and a spectrum geometrically scaled. In Sec. III, after analyzing the range of values for the strength of the effective interaction in terms of the particle masses, we illustrate the main results with examples. A general discussion on the applicability of the present approach and a summary of our conclusions are provided in Sec. IV.

\section{FORMALISM}

In this section, we present the basic formalism for a general charged three-body system, where the masses $\left(m_{1}, m_{2}, m_{3}\right)$ can be arbitrary, and the charges are such that we have a bound chargeless two-body subsystem $\left(q_{2}=-q_{1}\right)$ interacting with a slow charged particle $q_{3}$ (the charges are in units of the absolute value of the electron charge $|e|)$. The system Hamiltonian is given by

$$
\begin{gathered}
H=-\frac{\hbar^{2}}{2 \mu_{R}} \nabla_{R}^{2}-\frac{\hbar^{2}}{2 \mu_{r}} \nabla_{r}^{2}+V(\vec{r}, \vec{R}), \\
V(\vec{r}, \vec{R}) \equiv \frac{q_{1} q_{2}}{r}+\frac{q_{2} q_{3}}{\left|\frac{\mu_{r}}{m_{2}} \vec{r}+\vec{R}\right|}+\frac{q_{3} q_{1}}{\left|\frac{\mu_{r}}{m_{1}} \vec{r}-\vec{R}\right|},
\end{gathered}
$$

where $\vec{r}$ is the distance vector between the charges $q_{1}$ and $q_{2}$ and $\vec{R}$ the distance vector from the center of mass of the subsystem to the third (spectator) particle. Here, we define $\mu_{r}$ as the reduced mass of the subsystem $\left(q_{1} q_{2}\right)$, $\mu_{r} \equiv \mu_{12} \equiv\left(m_{1}^{-1}+m_{2}^{-1}\right)^{-1}$; and $\mu_{R}$ the corresponding reduced mass of the subsystem $\left(q_{1} q_{2}\right)$ interacting with the third particle: $\mu_{R} \equiv \mu_{(12) 3} \equiv\left[\left(m_{1}+m_{2}\right)^{-1}+m_{3}^{-1}\right]^{-1}$.

\section{A. Adiabatic approximation for three charges}

The adiabatic approximation implies in solving a coupled equation for the total wave function $\Psi(\vec{R}, \vec{r})=\psi(\vec{R}, \vec{r}) \Phi(\vec{R})$, such that we first solve the Schrödinger equation in the variable $\vec{r}$ for the wave function $\psi(\vec{R}, \vec{r})$, using $\vec{R}$ as a parameter, with energy solution $U(\vec{R})$,

$$
\left(-\frac{\hbar^{2}}{2 \mu_{r}} \nabla_{r}^{2}+V(\vec{r}, \vec{R})\right) \psi(\vec{R}, \vec{r})=U(\vec{R}) \psi(\vec{R}, \vec{r}) .
$$

Next, the total energy $E$ is given by

$$
\left(-\frac{\hbar^{2}}{2 \mu_{R}} \nabla_{R}^{2}+U(\vec{R})\right) \Phi(\vec{R})=E \Phi(\vec{R}) .
$$

The separation of the Schrödinger equation by means of the adiabatic approximation is justified in case the energy of the relative motion of the center of mass of the two-body subsystem, with respect to the third particle, is small in comparison with the subsystem binding energy.

Here, the main interest is the behavior of $U \equiv U(\vec{R})$ as $R$ goes to infinity. The case where the third particle interacts with the $\left(q_{1} q_{2}\right)$ subsystem. Using the expansion for the Coulomb potential, with $|\vec{R}| \gg|\vec{r}|$ and $P_{l}$ the usual Legendre polynomials of order $l$, we have

$$
\begin{aligned}
U \psi(\vec{R}, \vec{r})= & {\left[-\frac{\hbar^{2}}{2 \mu_{r}} \nabla_{r}^{2}+\frac{q_{1} q_{2}}{r}+\frac{q_{3}}{R} \sum_{l=0}\left\{q_{1}\left(\frac{-\mu_{r} r}{m_{1} R}\right)^{l}\right.\right.} \\
& \left.\left.+q_{2}\left(\frac{\mu_{r} r}{m_{2} R}\right)^{l}\right\} P_{l}(\hat{r} \cdot \hat{R})\right] \psi(\vec{R}, \vec{r}) .
\end{aligned}
$$

Assuming a neutral subsystem $\left(q_{1}=-q_{2}\right)$, the leading-order term (conformally invariant) in the asymptotic expansion $R \rightarrow \infty$ is given by

$$
U \psi(\vec{R}, \vec{r})=\left[-\frac{\hbar^{2}}{2 \mu_{r}} \nabla_{r}^{2}-\frac{q_{1}^{2}}{r}-\frac{q_{1} q_{3} r}{R^{2}} P_{1}(\cos \theta)\right] \psi(\vec{R}, \vec{r}),
$$

where $\theta$ is the angle between the vectors $\vec{r}$ and $\vec{R}$. The nextto-leading-order contribution to the adiabatic potential is $O\left(R^{-4}\right) P_{3}(\cos \theta)$ if $m_{1}=m_{2}$, otherwise a term like $O\left(R^{-3}\right) P_{2}(\cos \theta)$ appears, which however does not contribute due to parity conservation. Note that the higher-order terms in Eq. (5) break the conformal invariance.

The interaction between the third particle and the subsystem $\left(q_{1} q_{2}\right)$ at large distances is dominated by a dipole potential, which breaks the degenerated character of the opposite parity states. According to Ref. [3], there is an accumulation of resonances below the threshold of the $n=2$ excited state of the positronium negative ion, due to an attractive dipole interaction, which appears as the leading asymptotic term of the potential at large hyperspherical radius. Therefore, it is reasonable to assume that for large values of $R$ the first low lying levels of the $\left(q_{1} q_{2}\right)$-subsystem, i.e., the first-excited $S$ and $P$ states are weakly perturbed by the dipole interaction and the degeneracy is broken. For $R \rightarrow \infty$, the distorted wave function of the $\left(q_{1} q_{2}\right)$ subsystem is a linear combination of atomic orbitals (LCAOs), composed 
by $\psi(\vec{R}, \vec{r})=a(R) \phi_{(20)}(r) Y_{00}(\hat{r})+b(R) \phi_{(21)}(r) Y_{10}(\hat{r})$, where $Y_{l m}(\hat{r})$ (with $\left.l m=00,10\right)$ are the usual spherical harmonic function and $\phi_{n l}$ (with $n l=20,21$ ) are the two-body hydrogenic like wave functions for the charges $q_{1}$ and $-q_{1}$. Inserting $\psi(\vec{R}, \vec{r})$ in Eq. (6), we obtain

$$
\begin{gathered}
U(R)=-E_{1} \pm \frac{q_{1} q_{3}}{R^{2}}|K| \\
K \equiv \int d^{3} r \phi_{20}^{*}(r) Y_{00}^{*}(\hat{r}) r P_{1}(\cos \theta) \phi_{21}(r) Y_{10}(\hat{r}) \\
=-3\left(\hbar^{2} /\left(\mu_{r} q_{1}^{2}\right)\right),
\end{gathered}
$$

where $E_{1}$ is the energy of the first-excited state of the subsystem $\left(q_{1} q_{2}\right)$. The two possible signs in Eq. (7) come from the diagonalization of the eigenvalue Eq. (6) in the $2 S-2 P$ subspace.

From Eqs. (4), (7), and (8), we obtain the corresponding equation for the third particle motion related to the center of mass of the subsystem,

$$
\left(-\frac{\hbar^{2} \nabla_{R}^{2}}{2 \mu_{R}} \pm 3 \frac{\hbar^{2} q_{3}}{\mu_{r} q_{1}} \frac{1}{R^{2}}\right) \Phi=\left(E+E_{1}\right) \Phi \equiv \frac{\hbar^{2}}{2 \mu_{R}} \mathcal{E} \Phi .
$$

Here we note how the degeneracy between the $2 S$ and $2 P$ levels of the chargeless two-body subsystem is broken by the dipole potential. The motion of the wave function $\Phi \equiv \Phi(R)$ is given by an attractive type potential. The effect discussed in Ref. [4] of level condensation near the $N$ th state of positronium is analogous to this situation, as the longrange attractive potential $1 / R^{2}$ is responsible for forming such resonances. The other solution to $U(R)$ gives a longrange repulsion which means that, asymptotically, no bound states can be formed. This repulsive $1 / R^{2}$ interaction is responsible for the set of resonances above the $2 S-2 P$ states [5]. Focusing our approach in the weakly bound states of Eq. (9), we end up with an attractive dipole interaction, $\lambda / R^{2}$, generated by the particles with masses $m_{1}$ and $m_{2}$, where the dimensionless $\lambda$ is given by

$$
\lambda=6\left|\frac{q_{3}}{q_{1}}\right| \frac{\mu_{R}}{\mu_{r}}=6\left|\frac{q_{3}}{q_{1}}\right| \frac{\left(m_{1}^{-1}+m_{2}^{-1}\right)}{\left(m_{1}+m_{2}\right)^{-1}+m_{3}^{-1}} .
$$

Now, to solve Eq. (9), we employ a well-known result derived for attractive dipole potential (see [15]), which implies in a spectrum of infinite weakly bound levels $\mathcal{E}_{\nu}$ below $E=-E_{1}$, if $\lambda \geq 1 / 4$; otherwise, no other bound state. So, the following condition emerges for the existence of such spectrum:

$$
\frac{\left(m_{1}^{-1}+m_{2}^{-1}\right)}{\left(m_{1}+m_{2}\right)^{-1}+m_{3}^{-1}} \geq \frac{1}{24}\left|\frac{q_{1}}{q_{3}}\right| .
$$

In order to examine the condition suggested in [4] for the occurrence of level condensation, we restrict the above to an $A B A$ system with $\left|q_{B}\right|=\left|q_{A}\right|$ : If $m_{3}=m_{1}=m_{A}$ and $m_{2}=m_{B}$, we can easily verify that in all the cases we will have $\lambda \geq 1 / 4$, implying in a level spectrum below the state $n=2$ of the subsystem $(A B)$. The mass ratio $m_{A} / m_{B}$ in this case can only control the level spacing of the spectrum. However, if the bound subsystem is of a particle and antiparticle $\left(m_{A} \equiv m_{1}=m_{2}\right)$, with the captured particle having mass $m_{B}$, the condition $\lambda \geq 1 / 4$ will give us $m_{A} / m_{B} \leq 47.5$. This can be exemplified with the possible configurations of three-body systems with protons, electrons and their antiparticles, such as $\left(e^{-} e^{+}\right) p,\left(e^{-} e^{+}\right) \bar{p}$, and $(\bar{p} p) e^{ \pm}$, where level spectrum is expected to occur only for $\left(e^{-} e^{+}\right) p$ and $\left(e^{-} e^{+}\right) \bar{p}$.

\section{B. Geometrical scaling}

Equation (11) gives us a quite general relation which allows infinite number of energy levels that scale geometrically in most of three-body systems involving the usual stable charged particles and their antiparticles. The number of states are very dense when $\lambda \gg 1 / 4$, as the ratio between the levels is shown to be given by $[14,15]$ $\mathcal{E}_{\nu-1} / \mathcal{E}_{\nu}=\exp (2 \pi / \sqrt{\lambda-1 / 4})$, implying in the geometrically scaled energy levels

$$
\mathcal{E}_{\nu}=\mathcal{E}_{0} \exp (-2 \nu \pi / \sqrt{\lambda-1 / 4}),
$$

where $\mathcal{E}_{0}$ is a reference energy, solution of Eq. (9), determined by nonadiabatic effects. Observe that Eq. (9) is not well defined for small values of $R$ (of about few Bohr radii), leading to the collapse of the reference ground-state energy [15]. Therefore, the dipole potential must be modified at some short distance, which in our case corresponds to a region where nonadiabatic effects start to be relevant.

Two other important remarks related to Eqs. (10) and (11): (i) the strength of the dipole interaction, as well as the corresponding level ratio $\mathcal{E}_{\nu-1} / \mathcal{E}_{\nu}$ (when the occurrence of infinite number of levels is possible), depend only on the ratio of reduced masses $\mu_{(12) 3} / \mu_{12}$ and ratio of the charges $\left|q_{3} / q_{1}\right|$, implying that systems such as $\left(e^{-} e^{+}\right) e^{ \pm},\left(K^{-} K^{+}\right) K^{ \pm}$, or $(p \bar{p}) p$ have the same $\lambda$ and energy ratios $\mathcal{E}_{\nu-1} / \mathcal{E}_{\nu}$. (ii) Another relevant characteristic of Eq. (10) is its dependence on the specific three-body configuration and identification of the spectator particle: Let us consider two possible configurations for the same three-body system, where $m_{3} \ll m_{1}$ and $m_{3} \ll m_{2}$ and $q_{3}=q_{2}=-q_{1}$. With $\lambda \equiv \lambda_{(i j) k}$ for the configuration $\left(m_{i} m_{j}\right) m_{k}$, we obtain $\lambda_{(12) 3} \lambda_{(13) 2} \approx 36$. An obvious example is that of $\left(p e^{-}\right) \bar{p}$ or $\left(\bar{p} e^{+}\right) p$, where $\lambda \approx 3\left(m_{p} / m_{e}\right) \gg 1 / 4$ should lead to a dense level spectrum below the $n=2$ state of the subsystem. For the counterpart configuration, $(p \bar{p}) e^{ \pm}$, $\lambda=12\left(m_{e} / m_{p}\right) \ll 1 / 4$, a similar spectrum is not expected to occur.

\section{RESULTS AND EXAMPLES}

Figure 1 illustrates our conclusions for the values of $\lambda$ as a function of the mass ratios $m_{2} / m_{1}$ and $m_{3} / m_{1}$, taking $m_{2} \geq m_{1}$ and $\left|q_{3}\right|=\left|q_{1}\right|$. The critical value of $\lambda=1 / 4$ is shown by the dashed straight line. As observed, only in the cases that $24<m_{1} / m_{3}<47.5$ it is possible to reach the value $\lambda=1 / 4$ for some ratios of $m_{2} / m_{1}$. There is no infinite number of levels in case that $m_{1} / m_{3}>47.5$, independently of $m_{2} / m_{1}$. When $m_{1} / m_{3}=47.5$, the only solution is $m_{2}=m_{1}$; and, when $m_{1} / m_{3} \leq 24, \lambda>1 / 4$ for all choices of $m_{2}$ and $m_{1}$.

A few examples of three-body charged systems, with the corresponding values of $\lambda$ and level ratios $\mathcal{E}_{\nu-1} / \mathcal{E}_{\nu}$ are given 


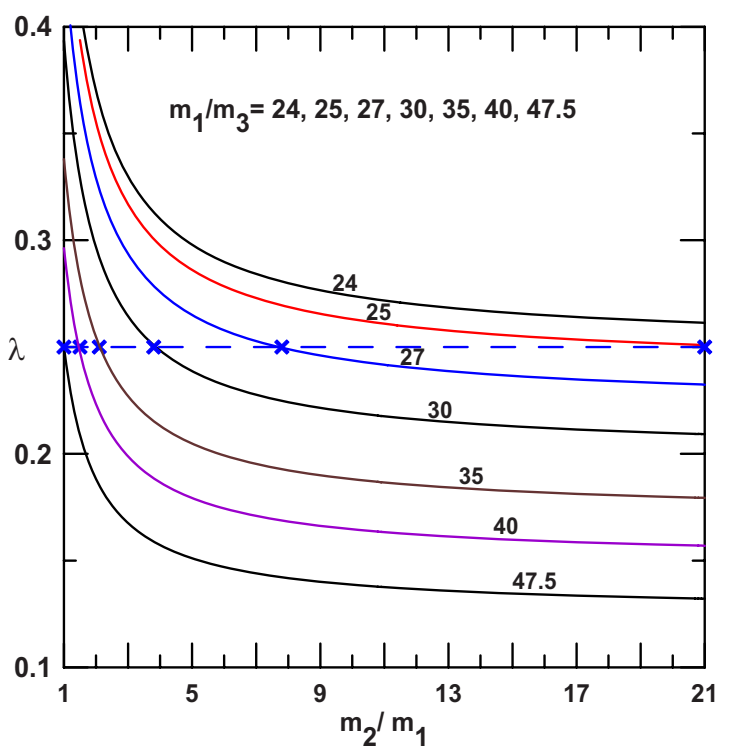

FIG. 1. (Color online) Behavior of $\lambda$ in terms of the ratio $m_{2} / m_{1}$. Each plot refers to a different ratio $m_{1} / m_{3}$, as given inside the figure. $\lambda>1 / 4$ is satisfied for all values of $m_{1}<24 m_{3}$; and $\lambda<1 / 4$ for all values of $m_{1}>47.5 m_{3}$.

in Table I. As verified, level condensation below the $n=2$ state of the subsystem (shown in the first two lines of the table) is not possible when the mass of the captured particle is much smaller than the lighter particle of the subsystem. In this table we also present a few results motivated by the actual interest in matter-antimatter interaction, and also motivated by experiments with exotic mesonic atoms [where protons $(p)$, deuterons $(d)$ or tritons $(t)$ are combined with

TABLE I. Strength $\lambda$ of the attractive dipole interaction (in dimensionless units) generated by the charges $q_{1}$ and $q_{2}=-q_{1}$, when capturing a charge $q_{3}$, for $\left|q_{3}\right|=\left|q_{1}\right|$ and different mass configurations. In the examples, the estimated value for $\lambda$ is the same for the cases with the corresponding antiparticle configurations. Estimations of level ratios $\mathcal{E}_{\nu-1} / \mathcal{E}_{\nu}$ are given when $\lambda>1 / 4$. The masses $m$ and $M$ are for arbitrary defined particles, with condition $M \gg m$.

\begin{tabular}{cccccc}
\hline \hline System & $\lambda$ & $\mathcal{E}_{\nu-1} / \mathcal{E}_{\nu}$ & System & $\lambda$ & $\mathcal{E}_{\nu-1} / \mathcal{E}_{\nu}$ \\
\hline$(p \bar{p}) e^{ \pm}$ & 0.0065 & & $\left(\mu^{+} \mu^{-}\right) e^{ \pm}$ & 0.058 & \\
$\left(K^{+} K^{-}\right) e^{ \pm}$ & 0.012 & & $\left(p \mu^{-}\right) e^{ \pm}$ & 0.025 & \\
$\left(m^{-} m^{+}\right) m^{ \pm}$ & 8 & 9.55 & $\left(M^{ \pm} m^{\mp}\right) m^{\mp}$ & 6 & 13.74 \\
$\left(e^{-} e^{+}\right) p$ & 24 & 3.63 & $\left(e^{-} e^{+}\right) \mu^{ \pm}$ & 23.75 & 3.66 \\
$\left(p \mu^{-}\right) \mu^{ \pm}$ & 6.06 & 13.55 & $\left(p K^{-}\right) K^{ \pm}$ & 6.81 & 11.63 \\
$\left(p e^{-}\right) p$ & 5508 & 1.09 & $\left(p e^{-}\right) \mu^{ \pm}$ & 1428 & 1.18 \\
$(p \bar{p}) \pi^{ \pm}$ & 1.66 & 198.62 & $(p \bar{p}) \mu^{ \pm}$ & 1.28 & 488.34 \\
$(p \bar{p}) K^{ \pm}$ & 5 & 17.87 & $(p \bar{p}) d$ & 12 & 6.25 \\
$(d \bar{d}) \mu^{ \pm}$ & 0.66 & $1.8 \times 10^{4}$ & $(t \bar{t}) \mu^{ \pm}$ & 0.44 & $1.8 \times 10^{6}$ \\
$\left(p \pi^{-}\right) p$ & 24.77 & 3.56 & $\left(p \mu^{-}\right) p$ & 31.22 & 3.09 \\
$\left(d \pi^{-}\right) d$ & 44.89 & 2.56 & $\left(d \mu^{-}\right) d$ & 57.82 & 2.29 \\
$\left(t \pi^{-}\right) t$ & 65.04 & 2.18 & $\left(t \mu^{-}\right) t$ & 84.45 & 1.98 \\
$\left(t \pi^{-}\right) p$ & 32.13 & 3.04 & $\left(t \mu^{-}\right) p$ & 41.84 & 2.65 \\
$\left(p \pi^{-}\right) t$ & 38.49 & 2.76 & $\left(p \mu^{-}\right) t$ & 48.11 & 2.48 \\
\hline \hline
\end{tabular}

muons $(\mu)$, kaons $(K)$ or pions $(\pi)]$. Our $\left(p \mu^{-}\right) p$ and $\left(d \mu^{-}\right) d$ results correspond to the ones given in [14]. Obviously, the same results apply, respectively, for $\left(p \mu^{-}\right) \bar{p}$ and $\left(d \mu^{-}\right) \bar{d}$. Table I displays the striking difference of such results when compared with the ones for $(p \bar{p}) \mu^{-}$and $(d \bar{d}) \mu^{-}$. Related to $\mathrm{Ps}^{-}$we should note that our approach gives $\lambda=8$, to be compared with $\lambda=7.06$ obtained by Botero [3] in a more involved calculation.

\section{DISCUSSION AND SUMMARY}

Before our conclusions, it is relevant a discussion on the applicability of the Born-Oppenheimer approximation to the particular configuration of excited states in three-charged systems. The separation of variables approximation, expressed in the assumption of a product form for the wave function of the three-body system, considered in Eq. (4), breaks down when the third particle gets close to the neutral subsystem, with $R$ of the order of the corresponding Bohr radius. For some three-body systems, as for example systems with proton and antiproton, nuclear force effects can also be relevant to be considered in addition to Coulomb effects. While in the asymptotic region the excited-state threecharged wave function can be described well by the BornOppenheimer approximation, as the size of the system gets smaller the magnitude of nonadiabatic effects is enhanced. In this case, in order to go beyond the adiabatic approximation, the appropriate description of the wave function will consist of an expansion with several higher-order terms, such that one cannot disregard the coupling between them. However, once one large three-charged state is formed with a size much larger than the dipole radius, i.e., with the wave function having major contribution from the asymptotic region, the nonadiabatic effects can be translated to a boundary condition on the wave function, at a radius characteristic of the region where nonadiabatic effects start to be important, which can be roughly estimated to be of the order of few Bohr radii. Within our present approximation we cannot obtain the first large bound excited state that spreads out in the asymptotic region of the dipole potential, as its energy is determined by nonadiabatic contributions. But, once this energy is known, the energies of the other large excited states follow the expression derived from the dipole interaction, emerging the geometrically scaled pattern given in Eq. (12).

In summary, considering a charged particle captured by a dimer (bound two-body subsystem), within an adiabatic approximation to the Coulombic three-body system with arbitrary masses, we derived the strength of the attractive dipole interaction with the critical condition for level condensation below the first-excited state of the dimer, as given in Eqs. (10) and (11). The adiabatic approach is justified when the energy of the relative motion of the dimer center of mass, with respect to the third particle, is small compared to the subsystem binding energy; and physically reasonable for a weak dipole potential interacting with the spectator particle. The particular straightforward charge-mass expression obtained for the leading term of the dipole interaction, as well as the condition to occur the geometrically scaled energy spectrum, can be very useful as an insight into the analysis of 
three-body charged systems with different mass relations. Of particular interest is the use of such approach to study interaction properties of matter and antimatter. Another relevant application can be found in the interaction of excitons with electrons in semiconductors [23]. As the electron and hole in the interacting pair of excitons can acquire effective individual masses distinct from the free-electron mass [24], general charge-mass conditions can be very helpful to analyze the relation between effective masses and the observed spectrum. We finally note that the present approach can be very useful to study capture reactions of a charged particle by a neutral two-body system, in view of possible three-body configurations. From a configuration without spectrum below the state $n=2$, one can generate a system with very dense spectrum by exchanging the spectator particle with the same charge particle of the neutral system. An exchange mechanism can change drastically the observed spectrum: for $\left(e^{-} e^{+}\right) p$ we have $\mathcal{E}_{\nu-1} / \mathcal{E}_{\nu}=3.63$ (levels below the first-excited state of Ps), whereas for $\left(e^{-} p\right) e^{+}$we have $\mathcal{E}_{\nu-1} / \mathcal{E}_{\nu}=13.74$ (levels below the first-excited state of the hydrogen atom).

\section{ACKNOWLEDGMENTS}

L.T. thanks Professor S. B. Duarte and Professor R. Shellard for the hospitality in CBPF. We acknowledge financial support from Fundação de Amparo à Pesquisa do Estado de São Paulo and Conselho Nacional de Desenvolvimento Científico e Tecnológico.
[1] J. A. Wheeler, Ann. N.Y. Acad. Sci. 48, 219 (1946).

[2] A. P. Mills, Jr., Phys. Rev. Lett. 46, 717 (1981).

[3] J. Botero and C. H. Greene, Phys. Rev. Lett. 56, 1366 (1986); J. Botero, Phys. Rev. A 35, 36 (1987).

[4] J. M. Rost and D. Wintgen, Phys. Rev. Lett. 69, 2499 (1992).

[5] Z. Papp, J. Darai, J. Zs. Mezei, Z. T. Hlousek, and C-.Y. Hu, Phys. Rev. Lett. 94, 143201 (2005).

[6] R. Krivec, M. I. Haftel, and V. B. Mandelzweig, Phys. Rev. A 47, 911 (1993); S. K. Adhikari, Phys. Lett. A 294, 308 (2002); G. W. F. Drake and M. Grigorescu, J. Phys. B 38, 3377 (2005); F. Fleischer et al., Phys. Rev. Lett. 96, 063401 (2006); M. Puchalski and A. Czarnecki, ibid. 101, 183001 (2008).

[7] W. Kolos, C. C. J. Roothaan, and R. A. Sack, Rev. Mod. Phys. 32, 178 (1960).

[8] C. L. Pekeris, Phys. Rev. 126, 1470 (1962); C. D. Lin, Phys. Rev. A 12, 493 (1975).

[9] A. A. Frost, M. Inokuti, and J. P. Lowe, J. Chem. Phys. 41, 482 (1964); R. K. Bhaduri and Y. Nogami, Phys. Rev. A 13, 1986 (1976); S. G. Lie, Y. Nogami, and M. A. Preston, ibid. 18, 787 (1978); A. Martin, J. M. Richard, and T. T. Wu, ibid. 46, 3697 (1992); R. A. Sultanov, ibid. 50, 2376 (1994); T. N. Rescigno et al., Science 286, 2474 (1999); V. I. Korobov, Phys. Rev. A 61, 064503 (2000); K. Varga, J. Usukura, and Y. Suzuki, Phys. Rev. Lett. 80, 1876 (1998); T. K. Rebane and A. V. Filinsky, Phys. At. Nucl. 65, 41 (2002); S. Takahashi and K. Takatsuka, J. Chem. Phys. 124, 144101 (2006); S. Paul, Eur. Phys. J. D 46, 483 (2008).

[10] C. D. Lin, Phys. Rep. 257, 1 (1995).

[11] E. A. G. Armour, J.-M. Richard, and K. Varga, Phys. Rep. 413, 1 (2005).
[12] D. R. Schultz, R. E. Olson, and C. O. Reinhold, J. Phys. B 24, 521 (1991).

[13] D. B. Cassidy and A. P. Mills, Jr., Nature (London) 449, 195 (2007).

[14] S. Kilic, J.-P. Karr, and L. Hilico, Phys. Rev. A 70, 042506 (2004).

[15] L. Landau and E. Lifchitz, Quantum Mechanics (MIR, Moscow, 1966); P. M. Morse and H. Feshbach, Methods of Theoretical Physics (McGraw-Hill, New York, 1953); see also, Yu. N. Ovchinnikov and I. M. Sigal, Ann. Phys. 123, 274 (1979).

[16] H. E. Camblong, L. N. Epele, H. Fanchiotti, and C. A. Garcia Canal, Phys. Rev. Lett. 87, 220402 (2001).

[17] E. Braaten and H.-W. Hammer, Phys. Rep. 428, 259 (2006).

[18] Z. Idziaszek, T. Calarco, P. S. Julienne, and A. Simoni, Phys. Rev. A 79, 010702(R) (2009).

[19] V. Efimov, Phys. Lett. B 33, 563 (1970).

[20] T. Kraemer et al., Nature (London) 440, 315 (2006).

[21] A. C. Fonseca, E. R. Redish, and P. E. Shanley, Nucl. Phys. A 320, 273 (1979).

[22] A. Delfino and T. Frederico, Phys. Rev. C 53, 62 (1996); A. Delfino, T. Frederico, and L. Tomio, Few-Body Syst. 28, 259 (2000).

[23] L. V. Butov et al., Nature (London) 417, 47 (2002); L. V. Butov, A. C. Gossard, and D. S. Chemla, ibid. 418, 751 (2002).

[24] M. Y. J. Tan, N. D. Drummond, and R. J. Needs, Phys. Rev. B 71, 033303 (2005).

[25] P. M. Platzman and A. P. Mills, Jr., Phys. Rev. B 49, 454 (1994).

[26] D. B. Cassidy et al., Phys. Rev. Lett. 95, 195006 (2005). 